\title{
Motifs temporels de la qualité de l'eau. Analyse d'une décennie de données haute-fréquence mesurées dans un observatoire agro-hydrologique*
}

\author{
Alice H. AUBERT ${ }^{1,2^{*}}$, Philippe MEROT ${ }^{1,2}$, Chantal GASCUEL-ODOUX ${ }^{1,2}$
}

${ }^{1}$ Inra, UMR 1069 Sol Agro et hydrosystème, Spatialisation, Rennes

${ }_{2}^{2}$ Agrocampus Ouest, UMR 1069 Sol Agro et hydrosystème, Spatialisation, Rennes. "e-mail: alice.helene.aubert@gmail.com

\begin{abstract}
RÉSUMÉ. - Le présent article est une synthèse de doctorat, portant sur l'analyse de données issues du bassin versant agricole Kervidy-Naizin, appartenant à l'Observatoire de Recherche en Environnement (ORE) AgrHys. Dès 2000, face aux enjeux de la qualité de l'eau et du changement climatique, le site a bénéficié d'un suivi hydrologique et chimique particulièrement fin. Les travaux présentés ci-après sont la première exploration de l'ensemble des chroniques longues de 12 ans du site de Kervidy-Naizin. Ces chroniques remarquables ont permis de répondre aux questions suivantes : quels sont les processus hydrologiques qui contrôlent les exportations de solutés dans le bassin versant ? Comment la dynamique temporelle d'humectation du bassin influence ces processus dominants ?

Quatre méthodes originales d'analyse fonctionnelle du bassin versant ont permis de répondre, en étudiant les trois niveaux de variabilité temporelle : la crue, la saisonnalité et les variations interannuelles. Ainsi, un modèle conceptuel d'exportation des solutés à quatre compartiments est proposé pour expliquer la diversité des dynamiques d'exportation dans ce bassin. Ces résultats montrent une structuration temporelle du signal de qualité de l'eau. Le signal, marqué pour certains solutés par l'activité agricole intensive, est dominé par la saisonnalité et varie sensiblement selon les conditions hydrométéorologiques de l'année, et notamment le changement d'état d'humidité du bassin.
\end{abstract}

Mots-clés : qualité de l'eau, chronique temporelle, observatoire long terme, variabilité temporelle, climat

\section{Temporal patterns of water quality: decadal high-frequency data-driven analysis from an hydrological observatory under agricultural land-use}

\begin{abstract}
The article synthetises PhD research on hydrological and hydrochemical data analyses from Kervidy-Naizin agricultural catchment (belonging to the Environmental Research Observatory AgrHys, itself part of the RBV network of French hydrological research catchments). Due to water quality and climate change issues, the catchment was heavily monitored as early as 2000 . The data-driven analyses presented hereafter consider for the first time the whole 12-year long dataset. Such outstanding time-series enabled to address the following questions: which hydrological processes are controlling solutes exports in the catchment? How does the wetness state dynamic influences these dominant processes?

Four original methods are used covering the range of temporal variability from flood event to seasonality and its inter-annual variations. A solute export conceptual model, composed of four hydrological compartments was proposed to explain the diversity of export dynamics for the catchment. Years presenting extrem hydro-meteorological conditions support this model, and emphasise on the importance of the change of catchment wetness state. Flood events also depend on that. Lastly, downslope advection and dispersion as well as riparian production, retention or mixing are also no negligible processes taking place in the catchment. Water quality signal is well structured in time and its structure is now further understood. The signal, strongly impacted by the agricultural use for given solutes, is dominated by seasonality but varies according to yearly hydrometeorological conditions. Comparing the solute export conceptual model built on Kervidy-Naizin to other catchments would be interesting to pinpoint driving factors.
\end{abstract}

Key-words: water quality, temporal data, long term observatory, temporal variability, climate

\footnotetext{
* Alice Aubert a obtenu le prix Henri Milon 2014 décerné par la SHF pour sa thèse « Analyse des motifs temporels d'une chronique décennale haute-fréquence de qualité de l'eau dans un observatoire agro-hydrologique; Méthodologies, typologies et processus ».
} 


\section{UN SITE D'ÉTUDE REMARQUABLE QUI PERMET UN QUESTIONNEMENT ORIGINAL}

\section{I.1. Le site de Kervidy-Naizin de l'Observatoire de Recherche en Environnement AgrHys}

L'ORE AgrHys a dès sa création en 1971 cherché à expliquer la qualité de l'eau de bassins versants consacrés à l'élevage, alors que l'agriculture s'intensifiait [Cheverry, 1998]. Les recherches qui y étaient et sont menées se placent dans le cadre de la définition de l'hydrologie qualitative donnée par l'Association Internationale des Sciences Hydrologiques dans l'article premier de ses statuts [AISH, 2011] : l'hydrologie qualitative est l'étude de la chimie de l'eau de rivière et de son rapport avec les conditions climatiques et le fonctionnement hydrologique du bassin versant. L'ORE a aujourd'hui les deux missions suivantes: (1) acquérir des données multi-variables de la qualité de l'eau dans différents compartiments hydrologiques pour comprendre les processus, les formaliser dans des modèles et (2) développer de nouvelles technologies pour le suivi de l'eau et de l'environnement. L'ORE se compose de deux sites, dont l'un est Kervidy-Naizin. Ce dernier est situé au cœur de la Bretagne, là où l'agriculture est intensive et l'élevage hors-sol très présent. En 2010, une enquête sur les pratiques agricoles a estimé le surplus total en azote à près de $200 \mathrm{~kg} \mathrm{~N} \mathrm{ha}^{-1}$ de surface agricole utile [Akkal, 2010].

En 2000, alors que 1'enjeu de la qualité de 1'eau est toujours d'actualité et que celui du changement climatique devient incontournable, le suivi de l'eau devient plus fréquent et couvre une vaste gamme de variables hydrologiques et hydrochimiques. En routine, les variables hydrologiques, météorologiques et hydrochimiques de la rivière sont mesurées au moins une fois par jour. Aussi, en 2010, après une décennie de suivi quotidien, complétée par des campagnes ponctuelles d'échantillonnage asservi aux crues et un suivi des nappes, un jeu de données d'une richesse remarquable était disponible, prêt à être analysé dans son ensemble.

De nombreux travaux ont porté sur l'étude du fonctionnement hydrologique de ce bassin versant. Ces travaux, servant à constituer les hypothèses de départ, portaient sur un élément (ou groupe d'éléments iso-chimiques) comme le nitrate [Molénat et al., 2005 ; Molénat et al., 2008], le COD [Lambert et al., 2011 ; Morel et al., 2009] ou encore sur les terres rares [Dia et al., 2000] (la liste des publications est loin d'être exhaustive).

\section{I.2. Recherche de motifs temporels}

Répondant à l'appel de Babovic [2005], qui se demande ce que peuvent nous apprendre toutes ces données issues d'observatoires à longs termes, suivi par Soulby et al. [2008], Gupta et al. [2008] et Hrachowitz et al. [2013], l'objectif est d'extraire plus d'informations des données pour développer et proposer de nouvelles théories, ou modèles conceptuels. Ces derniers pourront ensuite être transcrits en modèles numériques. Pour cela, Gupta et al. [2008] proposent de mettre l'accent sur la caractérisation de « signatures » qu'ils définissent comme des dynamiques de réponse, des comportements ou des motifs. Avant eux, Panu et al. [1978] avaient défini un "motif hydrologique » comme étant la description de données hydrologiques observées à pas de temps régulier.

\section{I.3. Étude de la variabilité temporelle}

La haute fréquence du suivi (au moins quotidien) et sa régularité sur plusieurs années sur l'ORE de Kervidy-Naizin permet de rechercher des motifs hydrologiques et hydrochimiques à différents pas de temps. En effet, dans la littérature, les études portant sur les effets du fonctionnement d'un bassin versant sur la qualité de l'eau se déclinent souvent en trois échelles temporelles: les tendances long terme, la saisonnalité et les réponses événementielles [Blöschl et Sivapalan, 1995 ; Burt et al., 2010 ; Moran et al., 2008]. Il s'agit alors de rechercher des motifs temporels de la qualité de l'eau pour les différents niveaux de variabilité et de caractériser les conditions hydrométéorologiques en lien avec ces motifs.

Après une description technique de l'instrumentation du site de Kervidy-Naizin, les motifs temporels seront présentés d'abord à l'échelle annuelle, et nous présenterons ensuite leurs variations inter-annuelles pour des conditions hydrométéorologiques particulières puis l'étude de motifs événementiels sera décrite. Enfin, les différentes échelles de temps seront considérées ensembles.

\section{INSTRUMENTATION DU SITE DE KERVIDY-NAIZIN}

La hauteur d'eau à la station de jaugeage (Fig.1) est mesurée à la minute (Thalimède OTT), la hauteur d'eau de la nappe tous les quarts d'heure le long de deux transects de 6 et 4 forages (Orpheus OTT).

L'eau de la rivière est prélevée manuellement tous les jours vers 17 heures, filtrée sur le champ (pores de $0,2 \mu \mathrm{m}$ ) puis conservée dans l'obscurité à $4^{\circ} \mathrm{C}$ dans des bouteilles de propylène remplies à ras bord. L'eau est analysée au plus tard 15 jours après le prélèvement. Les concentrations en anions majeurs (nitrate, sulfate, chlorure) sont mesurées par chromatographie ionique (DIONEX DX 100), avec une précision de $+-2,5 \%$. Les mesures de carbone sont réalisées avec un analyseur de carbone organique total (Shimadzu TOC 5050A), avec une précision de $5 \%$, évaluée à partir de 10 mesures sur des échantillons fraîchement préparés dans des solutions standard (K-phtalate).

La station agro-météorologique (Cimel Enerco 516i, identifiant Météo France : 56144001, dite « Le Toullo ») est située à un kilomètre de l'exutoire. Elle mesure le cumul des pluies sur une heure, les températures du sol à 10 et $50 \mathrm{~cm}$ de profondeur et de l'air à 10 et $50 \mathrm{~cm}$ de hauteur (moyenne horaire à partir de mesures toutes les 5 minutes), l'humidité de l'air, la vitesse du vent, le rayonnement global et la durée d'insolation (moyennes horaires).

Les données sont mises à disposition de la communauté scientifique dans la base de données AgrHys DataBase [http://geowww.agrocampus-ouest.fr/gene/agrhys/] et le portail géo-référencé Vidae [http://geowww.agrocampus-ouest. $\mathrm{fr} / \mathrm{web} /$ ?page_id=103].

\section{MOTIFS SAISONNIERS DE LA QUALITÉ DE L'EAU}

Définir une signature fonctionnelle chimique du bassin versant revient à définir la chronologie de la mobilisation des compartiments hydrologiques, spatialement distribués dans le bassin versant, ou en d'autres termes à identifier l'origine des solutés et les dynamiques internes et d'exportation des solutés. 


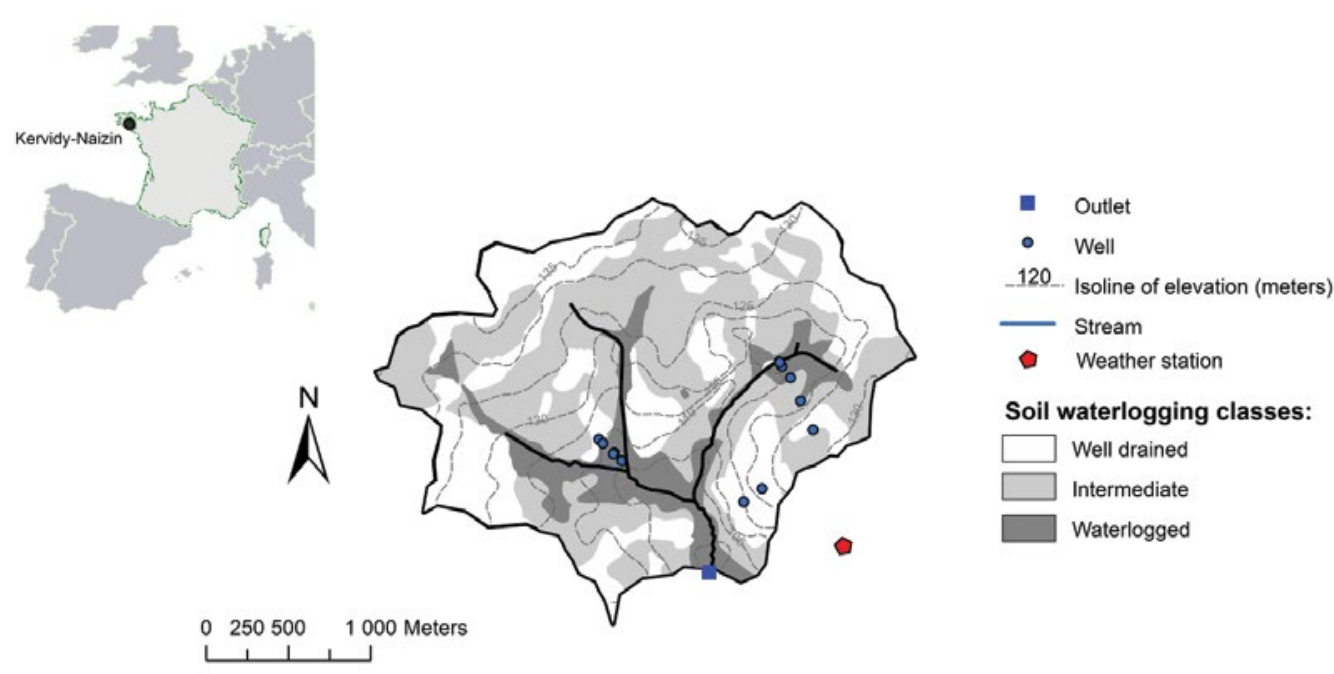

Figure 1 : Schéma du bassin versant de Kervidy-Naizin, localisation et instrumentation [Aubert et al., 2014, Supporting information].

Pour cela, l'ensemble des métriques suivantes a été étudié [Aubert et al., 2013a] : les concentrations moyennes en solutés dans quatre compartiments hydrologiques (rivière, nappe de versant, nappe superficielle de la zone humide et nappe profonde de bas de versant) définissant une signature dite « statique "; les variations annuelles (boîtes à moustache mensuelles et moyennes en crue et hors crues) et les corrélations temporelles interannuelles (variogrammes temporels des concentrations dans le cours d'eau) définissant une signature dite « temporelle ».

La signature «statique » permet de déduire l'origine des solutés dans le bassin versant, alors que la signature « temporelle » permet de décrire la dynamique des connexions des compartiments hydrologiques et les processus d'exportation.

$\mathrm{Au}$ cours de l'analyse des signatures statique et temporelle, un autre critère est apparu pour détailler la signature fonctionnelle du bassin versant : la période de constitution du stock de soluté, définie comme le temps entre l'entrée (ou la production) dans le bassin et l'exportation hors du bassin. Cette période peut n'être que la durée entre deux crues consécutives ou s'étendre sur plusieurs années.

Tous les solutés étudiés (nitrate, COD, chlorure et sulfate) montrent des variations saisonnières, mais les motifs de ces variations ne sont pas identiques, bien que deux groupes se distinguent : nitrate et chlorure d'une part et sulfate et carbone d'autre part.

La signature statique du nitrate et du chlorure se caractérise par des concentrations élevées dans la nappe de versant : ces solutés proviennent principalement des apports de fertilisants lixiviés et accumulés dans la nappe au fil des ans. Leur signature temporelle traduit un export saisonnier : quand la nappe de versant est mise en charge pendant la saison humide, elle est le contributeur principal aux écoulements. Ces signatures semblent caractéristiques de bassins versants agricoles où les niveaux d'intrants sont élevés et les processus hydrologiques dominés par la mise en charge d'une nappe superficielle de versant. La signature temporelle interannuelle du nitrate et du chlorure ressemble à celle du débit.

La signature statique du sulfate et du carbone diffère peu de celle observée dans des bassins versants moins impactés par l'Homme, comme par exemple le bassin de Plynlimon
[Reynolds et al., 1997]. Sulfate et chlorure sont plus concentrés en bas de versant que dans la nappe de versant (d'après leur signature statique) et proviennent principalement de la production bio-géo-chimique interne au bassin versant (d'après leur signature temporelle annuelle). Leur signature temporelle annuelle montre aussi des exportations dépendantes des crues témoignant d'une connexion de la zone humide à la rivière. La signature temporelle interannuelle du sulfate et du chlorure ressemble à celle de la température et de l'évapotranspiration.

Ces résultats permettent d'approfondir le concept de processus « contrôlés par le transport » [Creed et al., 1996 ; Hornberger et al., 1994 ; Martin et al., 2004]. En effet, ce sont les connexions des différents compartiments hydrologiques qui contrôlent le transport des solutés. Ces compartiments peuvent être des lieux de stockage des solutés (comme pour le nitrate et le chlorure) ou de production des solutés (comme pour le sulfate et le carbone). Les connexions des compartiments, et donc les exportations, ont lieu à l'échelle, soit annuelle (cas de la nappe de versant), soit événementielle (cas de la nappe superficielle de la zone humide). La variabilité des concentrations est maximale à la reprise des écoulements : les conditions hydrométéorologiques estivales influencent fortement les connexions, qu'elles soient saisonnières ou événementielles.

Cette classification fonctionnelle (Fig.2) pourrait être utilisée dans le cadre de l'hydrologie comparative.

\section{VARIATIONS INTERANNUELLES DES MOTIFS SAISONNIERS}

Après avoir défini les motifs saisonniers de la qualité de l'eau dans le bassin versant de Kervidy-Naizin, les variations interannuelles de ces motifs liées aux conditions météorologiques ont été étudiées à leur tour. La décennie de données de Kervidy-Naizin le permet car, parmi les 10 années, 2000-01 est une année très humide, 2004-05 très sèche (observation basée sur les débits et la pluviométrie) et l'été de 2003 très chaud.

La méthode suivante est proposée [Aubert et al., 2013b] : représenter les moyennes mensuelles des concentrations 


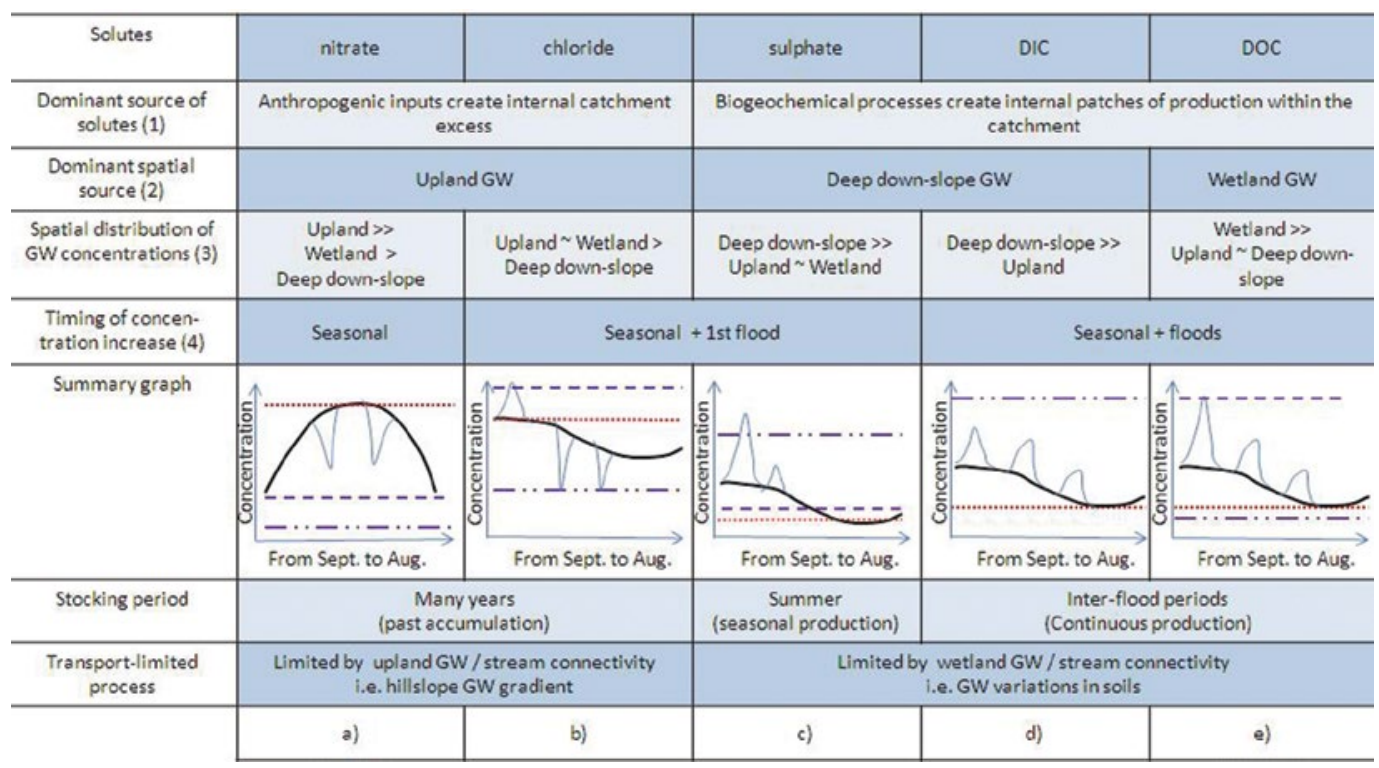

Figure 2 : Classification des motifs temporels des solutés et de leurs déterminants [Aubert et al., 2013a].

dans la rivière en fonction des moyennes mensuelles d'une variable hydrométéorologique choisie. Cette méthode permet de garder une dimension temporelle et de comparer les années entre elles.

Afin d'identifier la variable hydrométéorologique d'intérêt, une première phase a porté sur l'étude de la relation entre le débit et la température de l'air (linéairement corrélée à la température de l'eau sous le climat breton). Cette relation constitue une signature hydrométéorologique du bassin versant. Dans le cas de Kervidy-Naizin, la moyenne mensuelle des moyennes glissantes sur une fenêtre de 30 jours des températures est linéairement corrélée avec le débit. Ceci appuie le rôle de l'évapotranspiration comme facteur contrôlant la saisonnalité hydrologique dans le bassin versant.

La seconde phase a porté sur la relation entre la variable hydrométéorologique et les concentrations en solutés. Dans tous les cas, la représentation des données a conduit à analyser des hystérèses (Fig.3), traduisant un décalage temporel
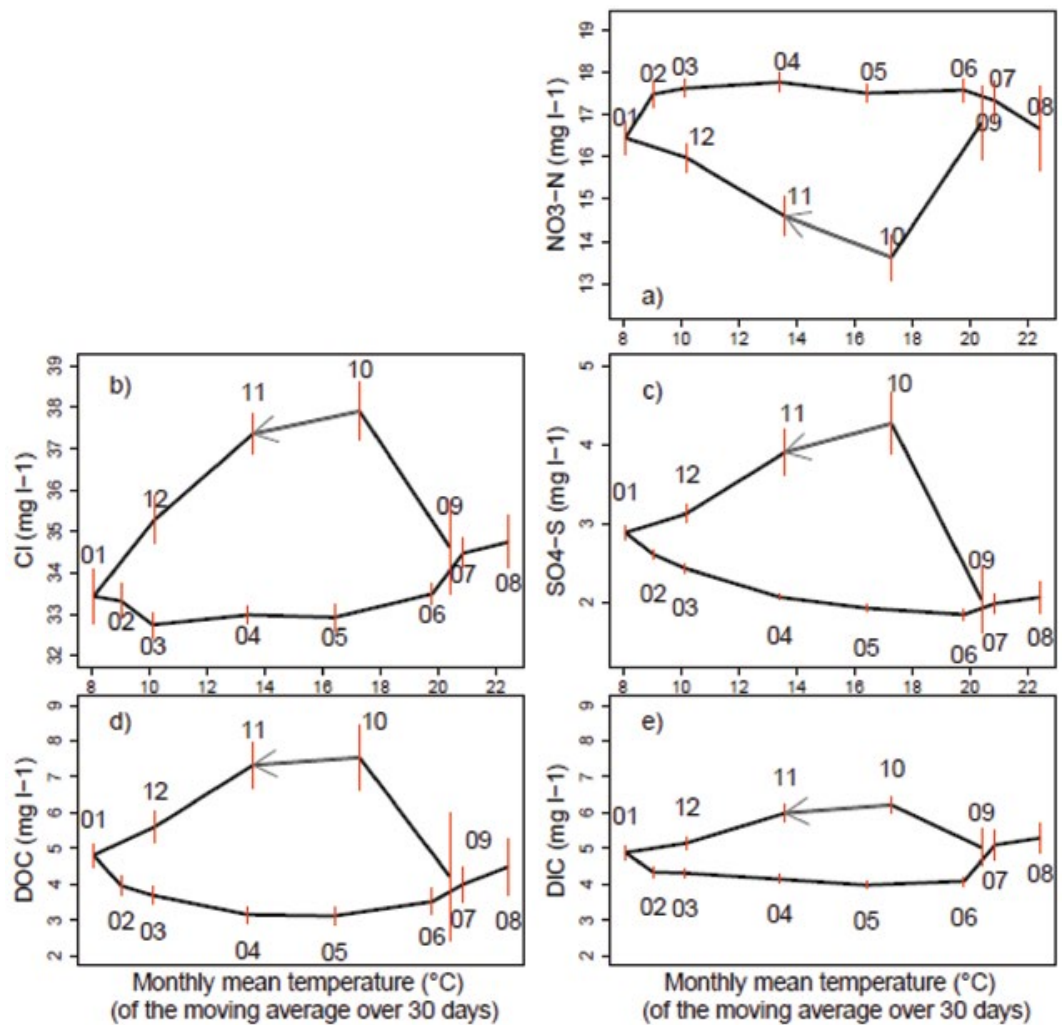

Figure 3 : Moyennes mensuelles des concentrations en (a) nitrate- $N$, (b) chlorure, (c) sulfate-S, (d) COD et (e) CID (mg. $\left.l^{-1}\right)$ en fonction des moyennes mensuelles des moyennes glissantes sur une fenêtre de 30 jours des températures. L'hystérèse est horaire pour le nitrate, anti-horaire pour les autres solutés. Les barres d'erreur représentent les intervalles de confiance [Aubert et al., 2013b]. 
entre les variations des solutés et celles des conditions hydrométéorologiques. Seuls le nitrate se distingue avec un cycle inversé par rapport aux autres éléments. Aussi, le sens de l'hystérèse peut s'expliquer par le fonctionnement hydrologique du bassin versant : les solutés dont le compartiment de stockage (constitué ou généré pendant l'été) est proche de la rivière et rapidement connecté à la reprise des écoulements, présentent des hystérèses dans un sens (anti horaire avec les températures), alors que les solutés dont le compartiment le plus concentré a besoin de temps pour se connecter (temps d'humectation du bassin) présentent des hystérèses inversées (horaire avec les températures). Les hystérèses se composent de trois domaines, appuyant l'existence de trois périodes hydrologiques.

\section{MOTIFS ÉVÉNEMENTIELS DE LA QUALITÉ DE L'EAU}

Les variations de la qualité de l'eau au cours des crues et leur variabilité constituent un autre niveau d'étude. La méthode d'exploration des données (data mining) choisie utilise le formalisme Latent Dirichlet Allocation (LDA) et il s'agit, à notre connaissance, de sa première application dans le domaine de l'hydrologie [Aubert et al., 2013c]. Ce formalisme recherche les co-occurrences de données les plus probables sans limitation sur les nombres de variables. Le résultat obtenu est un ensemble de motifs (autant que demandés) représentant les co-occurrences les plus probables, c'est-à-dire l'observation de telle donnée en même temps que telle autre. Grâce à cette méthode, les crues ne sont pas décrites par des variables intermédiaires (concentration maximale, ...). Il faut cependant définir des classes de valeurs pour les différentes variables.

L'algorithme LDA est utilisé pour partitionner les crues en fonction des variations de la chimie de l'eau : d'après les études préalables, un certain nombre d'hypothèses sur ces variations en crue ont été formulées. Par exemple, on s'attend à des pics de COD lors des crues et des dilutions pour le nitrate. On suppose également des signatures de crues différentes en fonction de la période hydrologique. Il s'agit de tester ces hypothèses.

Trois motifs de crues ont été obtenus, basés sur les co-occurrences de données les plus probables. Ces motifs sont très clairement distribués dans le temps : chaque motif est spécifique d'une période hydrologique donnée. Par exemple (Fig.4), le motif caractérisé par une faible dilution de concentrations élevées en chlorure, un pic positif des concentrations en $\mathrm{COD}$ et sulfate et une légère dilution des concentrations en nitrate est plus probable en octobre et novembre, dans un contexte hydrologique d'augmentation des débits et niveaux de la nappe, ce qui correspond à la période de reprise des écoulements. Les conditions hydrométéorologiques de l'année ont aussi un effet sur la distribution interannuelle des motifs de crues, même si cela est moins marqué : un motif particulier a été généré pour l'année la plus sèche (2004-05).

Cette méthode semble prometteuse dans le domaine de l'hydrologie, aussi bien quantitative que qualitative. Il serait intéressant de la voir appliquer dans d'autres études. Elle permet de traiter d'un grand nombre de données, ce qui devient de plus en plus fréquent.

\section{STRUCTURE DE LA CHRONIQUE TEMPORELLE À DIFFÉRENTES ÉCHELLES DE TEMPS}

Après avoir défini les variations de la qualité de l'eau aux niveaux saisonnier et événementiel (en comparant les années), une méthode d'analyse du signal, l'analyse spectrale, a été appliquée sur le jeu de données. L'analyse (a) Pattern 0

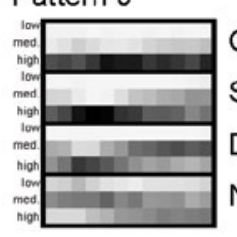

Chloride concentration

Sulfate concentration

DOC concentration

Nitrate concentration

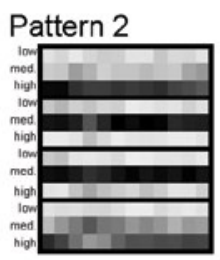

Chloride concentration

Sulfate concentration

DOC concentration

Nitrate concentration

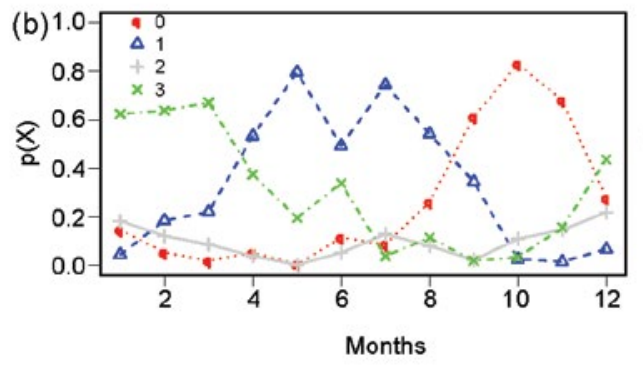

Pattern 1

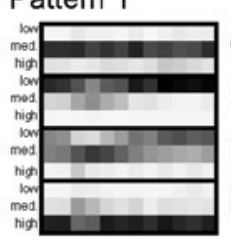

Chloride concentration

Sulfate concentration

DOC concentration

Nitrate concentration

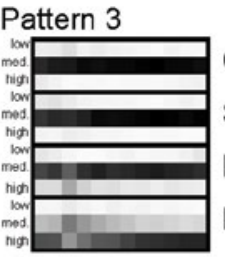

Chloride concentration

Sulfate concentration

DOC concentration

Nitrate concentration

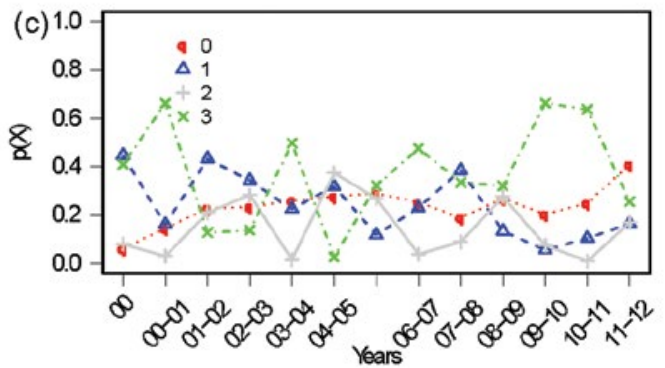

Figure 4 : Motifs temporels des solutés au cours d'une crue et de leur répartition mensuelle et interannuelle [Aubert et al., $2013 c]$. 
spectrale caractérise la variabilité de la chronique pour différentes échelles temporelles. Elle donne une mesure de la variance d'une chronique dans un continuum d'échelle de temps exprimé dans le domaine fréquentiel. Elle a été utilisée dès les années 60 [par exemple: Gunnerson, 1966 ; Thomann, 1967] pour (i) identifier les périodicités d'une chronique et estimer la variance pour les cycles de cette période, (ii) pour caractériser la structure d'une chronique peu périodique (observe-t-on un bruit blanc, un bruit brownien ou fractal ?), (iii) valider un modèle en comparant la structure de la chronique simulée à celle de la chronique observée et (iv) calculer des fonctions de transfert, à condition d'avoir des chroniques d'entrée et de sortie.

L'analyse spectrale est ici utilisée pour caractériser la structure du signal pour une trentaine d'éléments chimiques afin de détecter les propriétés sous-jacentes aux chroniques, approfondir la connaissance de la périodicité interannuelle et aborder la variabilité sub-journalière [Aubert et al., 2014]. Le jeu de données quotidiennes en anions majeurs et COD est enrichi de trois ans d'échantillonnage journalier pour une trentaine d'éléments (ICP-MS) et une année d'échantillonnage toutes les 20 minutes pour le nitrate et le COD (spectrophotomètre UV-visible).

D'un point de vue de la structure du signal, nous retrouvons ce que Blöschl et Sivapalan (1995) ont qualifié de typique en hydrologie, à savoir que les processus les plus longs ou basse fréquence présentent une puissance spectrale (grandeur proportionnelle à la variance) plus élevée que les processus plus brefs.

Pour les cycles plus longs que les cycles journaliers, le signal est de type fractal alors que les dynamiques de transfert des éléments étudiés sont de deux types : (i) soit l'exportation est contrôlée par la dynamique saisonnière de la nappe de versant, (ii) soit l'exportation est contrôlée par une dynamique événementielle, les éléments étant dans l'horizon superficiel du sol. La périodicité annuelle, seul cycle observé dans les données, confirme ces deux dynamiques : elle explique presque la moitié de la variance de la chronique dans le premier cas, et est presque nulle dans le second cas. L'universalité du signal fractal doit s'expliquer par un processus qui dépasse ces différences de dynamique d'export. Une hypothèse avancée, et vérifiée d'un point de vue théorique [Kirchner et Neal, 2013], est l'existence d'advection et de dispersion en bas de versant, voire dans le cours d'eau. Ces processus atténuent la puissance spectrale des variations les plus brèves.

Pour les très hautes fréquences (cycles inférieurs à 3 heures), le signal tend vers du bruit blanc (Fig.5), identifié comme du bruit de mesure, bien que cela reste à vérifier avec les caractéristiques spectrales de l'appareil de mesure.

Il serait intéressant de comparer maintenant la structure des chroniques simulées par le modèle de bassin versant TNT2 développé sur Kervidy-Naizin.

\section{CONCLUSION GÉNÉRALE}

\section{VII.1. Une structuration spatio-temporelle des chroniques de qualité de l'eau}

L'ensemble des travaux présentés ici valorise les données de longs termes mesurées sur le bassin versant de Kervidy-Naizin de l'ORE AgrHys, représentatif des bassins versants sur socle imperméable, en milieu tempéré, dont l'hydrologie est dominée par la fluctuation de la nappe, dans un contexte où l'activité agricole (notamment
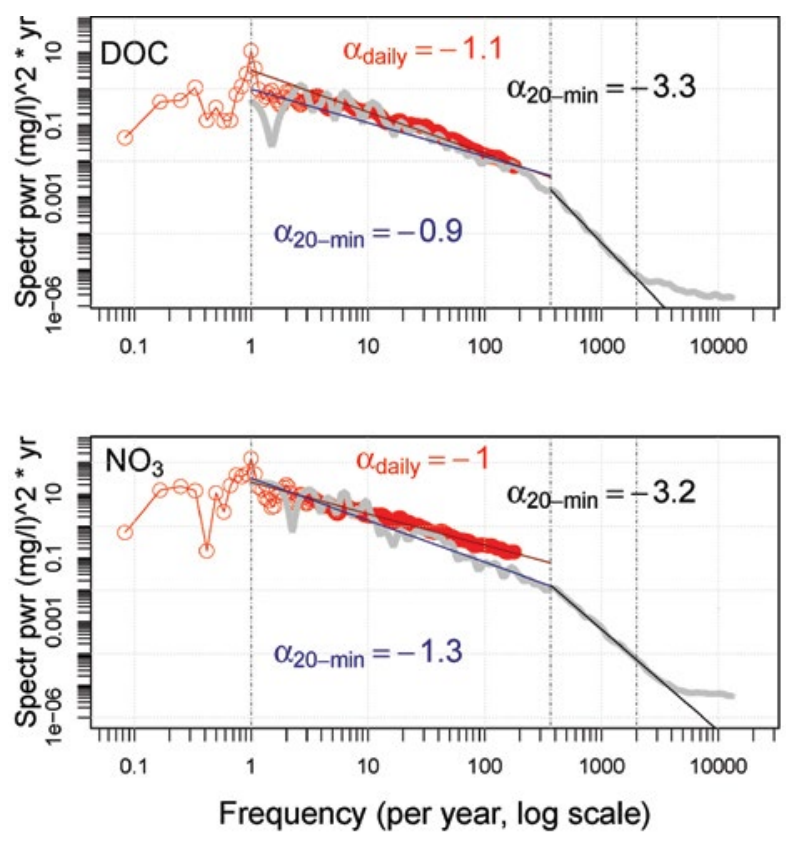

Figure 5 : Densité de puissance spectrale pour le nitrate et le DOC [Aubert et al., 2014].

l'élevage hors-sol) est intensive. Cette valorisation permet de comprendre de façon plus systémique le fonctionnement du bassin versant, grâce à l'étude de la saisonnalité et de ses variations interannuelles, de la typologie des crues et de l'analyse de la structure des chroniques dans le domaine fréquentiel.

Les variations des concentrations en solutés sont dominées par la saisonnalité, y compris pour les variations événementielles. Cette saisonnalité varie sensiblement en fonction des conditions hydro-météorologiques de l'année, mais trois périodes hydrologiques se retrouvent : la période de hautes eaux, la période de transition vers l'étiage estival et la période de reprise des écoulements. Cette dernière est la plus variable, en partie parce que ce cycle hydrologique est parfois interrompu par une période sans écoulement. Cette possible interruption semble due aux conditions météorologiques de l'année, en particulier à l'évapotranspiration. En effet, l'évapotranspiration joue sur le dessèchement estival et donc sur la dynamique de réhumectation du bassin versant à l'automne. Le dessèchement estival et les pluies efficaces automnales contrôlent la connexion des quatre compartiments hydrologiques, contributeurs aux écoulements, chacun ayant une signature chimique qui lui est propre.

A ces périodes hydrologiques correspondent des périodes hydrochimiques, c'est à dire, à chaque saison il y a une signature de la chimie de l'eau différente. Ces signatures hydrochimiques s'expliquent par les contributions variables au cours du temps de différents compartiments hydrologiques, chacun étant caractérisé par des conditions particulières et donc des niveaux de concentrations différents.

Ce motif de distribution spatiale des concentrations en quatre compartiments est lié à la géologie et à la pédologie du bassin versant (distribution des horizons organiques dans les sols, profondeur du socle imperméable, présence de pyrite dénitrifiante), à l'activité agricole intensive (la fertilisation conduit à des concentrations en nitrate et chlorure élevées) et aux structures du paysage (zone humide pouvant être dénitrifiante). 


\section{VII.2. Des contributions méthodologiques}

Cette compréhension du fonctionnement du bassin versant a été possible après avoir identifié des motifs temporels de la qualité de l'eau ou des signatures hydrochimiques grâce à quatre méthodes. Chacune a été utilisée pour étudier un niveau de variabilité temporelle donné, mais toutes pourraient servir/servent déjà à étudier d'autres niveaux de variabilité temporelle de la qualité de l'eau ou du débit.

Les méthodes proposées sont aussi transposables à d'autres contextes, d'autres jeux de données temporels hydrologiques et hydrochimiques. Il faudrait rechercher dans quelles mesures les motifs obtenus sur Kervidy-Naizin se retrouvent dans d'autres bassins versants, de tailles et de contextes géopédoclimatiques différents.

La richesse du jeu de données disponible sur l'ORE AgrHys garantit qu'il est encore possible de faire des avancées en termes de méthodes d'analyse de données temporelles. Des méthodes d'analyse multivariées auraient aussi pu être utilisées. Il serait souhaitable d'explorer plus finement la période de reprise des écoulements qui est très variable, ceci en s'attachant aux données des années hydrologiques où la reprise des écoulements est soit précoce soit tardive.

\section{REMERCIEMENTS}

Nous remercions tout le personnel de 1'UMR Géosciences (CNRS, Université de Rennes 1) et de 1'UMR Sol, Agro et hydrosystème, Spatialisation (Inra, Agrocampus Ouest) travaillant sur le terrain et au laboratoire qui a permis d'avoir aujourd'hui ce jeu de données, notamment : Martine Lecoz-Boutnik, Patricia Madec, Patrice Petitjean, Mikael Faucheux, Yannick Fauvel et Yannick Hamon, ainsi que Jean-Paul Guillard qui prélève chaque jour l'eau du cours d'eau. Nous remercions l'Inra et le CNRS pour leur soutien au dispositif RBV (SOERE) dans lequel s'inscrit AgrHys, l'ANR pour le financement des différents programmes de recherche accueillis sur les sites.

\section{REFERENCES}

AKKal N. (2010) - Description des systèmes d'exploitation du bassin versant de Kervidy-Naizin. NitroEurope IP 71

AISH/IAHS (2011) — Statutes and bye-laws of IAHS revised July 2011. http://iahs.info/About-IAHS/Statutes-En.do, consulté le 24.06.2014

Aubert A.H., Gascuel-Odoux C., Gruau G., et Al. (2013) Solute transport dynamics in small, shallow groundwater-dominated agricultural catchments: Insights from a high-frequency, multisolute 10 yr-long monitoring study. Hydrology and Earth System Sciences 17(4) 1379-1391

Aubert A.H., Gascuel-Odoux C., Merot P. (2013) - Annual hysteresis of water quality: A method to analyse the effect of intraand inter-annual climatic conditions. Journal of Hydrology 478 29-39

Aubert A.H., Tavenard R., Emonet R., ET AL. (2013) Clustering flood events from water quality time series using Latent Dirichlet Allocation model. Water Resources Research 49 8187-8199

Aubert A.H., Kirchner J.W., Gascuel-Odoux C., ET AL. (2014) Fractal Water Quality Fluctuations Spanning the Periodic Table in an Intensively Farmed Watershed. Environmental Science \& Technology 48 930-937
BABovic V. (2005) - Data mining in hydrology. Hydrological Processes 19(7) 1511-1515

Bloschl G., Sivapalan M. (1995) - Scale issues in hydrological modeling - a review. Hydrological Processes 9(3-4) 251-290

Burt T.P., Howden N.J.K., Worrall F., ET AL. (2010) — Long-term monitoring of river water nitrate: how much data do we need? J. Environ. Monit 12(1) 71-79

CheVerry C. (1998) - Agriculture intensive et qualité des eaux. Paris, INRA éditions.

Creed I.F., Band L.E., Foster N.W., ET AL. (1996) - Regulation of Nitrate-N Release from Temperate Forests: A Test of the N Flushing Hypothesis. Water Resources Research 32 3337-3354

Dia A., Gruau G., Olivie-Lauquet G., et al. (2000) - The distribution of rare earth elements in groundwaters: Assessing the role of source-rock composition, redox changes and colloidal particles. Geochimica Et Cosmochimica Acta 64(24) 4131-4151

GuNNERSON C.G (1966) — Optimizing sampling intervals in tidal estuaries. Sanit. Eng. Div. ASCE, 92(SA2) 103-125

GuPTA H.V., Wagener T., LiU Y.Q. (2008) — Reconciling theory with observations: elements of a diagnostic approach to model evaluation. Hydrological Processes 22(18) 3802-3813

Hornberger G.M., Bencala K.E., Mcknight D.M. (1994) Hydrological controls on dissolved organic carbon during snowmelt in the Snake River near Montezuma. Colorado. Biogeochemistry 25 147-165

Hrachowitz M., SaveniJe H.H.G., Blöschl G., ET Al. (2013) - A decade of Predictions in Ungauged Basins (PUB) - a review. Hydrological Sciences Journal 58(6) 1198-1255. http://www. tandfonline.com/doi/pdf/10.1080/02626667.2013.803183

Lambert T., Pierson-Wickmann A.-C., Gruau G., et Al. (2011) - Carbon isotopes as tracers of dissolved organic carbon sources and water pathways in headwater catchments. Journal of Hydrology 402(3-4) 228-238

Martin C., Aquilina L., Gascuel-Odoux C., et Al. (2004) Seasonal and interannual variations of nitrate and chloride in stream waters related to spatial and temporal patterns of groundwater concentrations in agricultural catchments. Hydrological Processes 18(7) 1237-1254

Molenat J., Gascuel-Odoux C., Davy P., et Al. (2005) - How to model shallow water-table depth variations: the case of the Kervidy-Naizin catchment, France. Hydrological Processes 19(4) $901-920$

Molenat J., Gascuel-Odoux C., Ruiz L., et Al. (2008) - Role of water table dynamics on stream nitrate export and concentration. in agricultural headwater catchment (France). Journal of Hydrology 348(3-4) 363-378

Moran M.S., Peters D.P.C., Mcclaran M.P., et Al. (2008) Long-term data collection at USDA experimental sites for studies of ecohydrology. Ecohydrology 1(4) 377-393

Morel B., Durand P., JafFrezic A., ET AL. (2009) - Sources of dissolved organic carbon during stormflow in a headwater agricultural catchment. Hydrological Processes 23(20) 2888-2901

Panu U.S., UnNy T.E., Ragade R.K. (1978) - Feature prediction model in synthetic hydrology based on concepts of pattern-recognition. Water Resources Research 14(2) 335-344

Reynolds B., Renshaw M., Sparks, et al. (1997) — Trends and seasonality in stream water chemistry in two moorland catchments of Upper River Wye, Plynlimon. Hydrology and Earth System Sciences 1 571-581

Soulsby C., Neal C., Laudon H., et AL. (2008) - Catchment data for process conceptualization: simply not enough? Hydrological Processes 22(12) 2057-2061

Thomann R.V. (1967) — Time series analysis of water quality data. J. Sanit. Eng. Div. ASCE 93(SA1) 1-23 